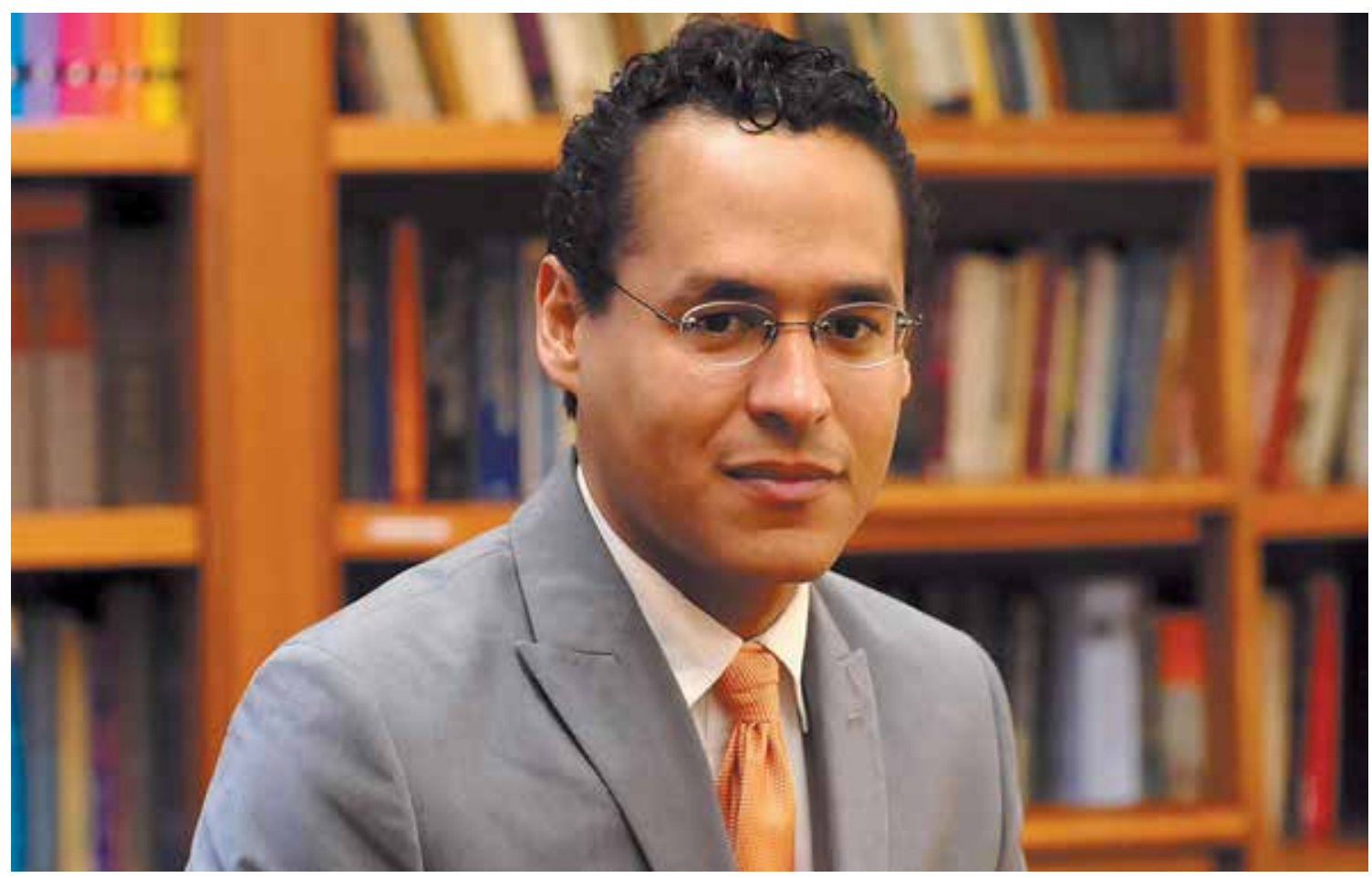

\title{
Entrevista a Roosevelt Montás, director del Core Curriculum de la Universidad de Columbia
}

\author{
"Yo mismo estudié a nivel de grado en la Universidad de Columbia, habiendo emigrado a \\ Estados Unidos a la edad de 12 años y este curriculum, esta educación general, clásica, \\ transformó literalmente mi vida y me llevó a la carrera como académico, profesor y ahora \\ director del mismo programa".
}

En su experiencia como egresado y académico de un currículum de Estudios Generales y del cual hoy es su director, ¿cuáles son los fundamentos de estos estudios y cómo podría aclararnos la diversidad terminológica que se utiliza para nombrarlos?

Soy Roosevelt Montás de la Universidad de Columbia. Les agradezco la oportunidad de dirigirme a todos ustedes desde Nueva York.

Lo que voy a presentar en esta entrevista es un sondeo general de los fundamentos y propósitos del estudio de las artes liberales; también quiero describir al final el modelo de educación liberal que utilizamos en la Universidad de Columbia en torno al "Core Curriculum", que es el programa de estudios liberales que tengo el honor de dirigir. Creo que primero debo comenzar por clarificar mis términos. A veces se usa el término "educación general" para describir aquella parte de la educación de un alumno universitario que no es específica de su carrera y, por lo tanto, la toman en común $-\mathrm{y}$ a veces juntos- con otros estudiantes de diferentes carreras. Un término que a veces se intercambia con el de la "educación general" es el término "educación liberal" o "educación en artes liberales", a veces simplemente se habla de "las humanidades". Entre estos términos, artes liberales es el más amplio y a la vez el más específico; es también el término de mayor uso aquí en los Estados Unidos. Así que, ese es el término que voy a utilizar, pero entiéndase que uso el término "artes liberales" en forma intercambiable con el término estudios generales.

Otra aclaración en cuanto a los términos es el adjetivo "liberal", el cual tiene un sentido de corriente política y se contrasta con el término "conservador", pero ese no es el sentido que utilizamos cuando aplicamos el término "liberal" a la educación. En el ámbito académico, la educación liberal se contrasta con la educación técnica, o con la educación profesional, o 
con la educación vocacional. La educación liberal es un tipo de educación que no está dirigida a ningún oficio específico, sino que se enfoca en el desarrollo humano general, siempre con vista a indagar sobre el significado de la vida humana y sobre cuestiones existenciales que son la herencia común de todos los seres conscientes, o sea, de los seres que son conscientes de su vida, y, a la vez, de su mortalidad; así que, lo que caracteriza la educación liberal es que se enfoca en los fines de la vida humana, en vez de los medios para lograr uno u otro fin. Si, por ejemplo, la ingeniería se pregunta: “¿cómo se hace un puente?”; la educación liberal se pregunta: “¿por qué se hace un puente?”, y “¿cuál es el valor de un puente?", y, ¿se debe hacer un puente en estas circunstancias, aquí y ahora? Sus preguntas y preocupaciones tienen que ver con los fundamentos del bien humano y con relación a los cuales la vida adquiere su significado. Las preguntas que animan la educación general son las preguntas de un ser libre.

\section{¿Cuál es el origen o pertinencia de los estudios liberales?}

Para dar foco a la pertinencia de los estudios liberales permítanme presentarles un antiguo relato budista. El desarrollo del relato es que el rey Pasenadi de Cósala se presenta ante Buda y Buda le pregunta: “¿Qué has estado haciendo?". Y el rey le responde: "ya sabes, haciendo cosas de reyes, asuntos de estado, oficios de ese tipo". Entonces Buda le dice lo siguiente: ¿Qué piensas, gran rey? Supongamos que un hombre creíble, confiable, viniera ante ti desde el este y al llegar ante ti dijera: "Si le place, majestad, debe saber que vengo desde el este y allí vi una gran montaña del alto de las nubes que viene hacia nosotros aplastando a todo ser viviente; haga usted lo que crea que debe de hacerse". Entonces un segundo hombre también confiable y creíble viene desde el oeste y le dijera: "Si le place, majestad, usted debe saber que vengo desde el oeste y allí vi una gran montaña de lo alto de las nubes que viene hacia nosotros aplastando a todo ser viviente; haga usted lo que crea que debe de hacerse". Llega entonces un tercer hombre que viene desde el norte y le dice: "Si le place, majestad, debe saber que vengo desde el norte y allí vi una gran montaña del alto de las nubes que viene hacia nosotros aplastando a todo ser viviente; haga usted lo que crea que debe de hacerse". Y finalmente, llegó un hombre desde el sur y le dice: "Si le place, majestad, usted debe saber que vengo desde el sur y allí vi una gran montaña del alto de las nubes que viene hacia nosotros aplastando a todo ser viviente; haga usted lo que crea que debe de hacerse".

$\mathrm{Si}$, gran rey, un peligro tan grande surgiese, tal terrible destrucción de la vida humana -la condición humanasiendo tan difícil de alcanzar ¿qué debe hacerse, gran rey? A esto el rey le responde: "Sí, señor, si tal peligro surgiese, tan terrible destrucción de la vida humana -la condición humana siendo tal difícil de alcanzar¿qué debe hacerse, sino la conducta de Dhamma, la conducta correcta, los hechos meritorios?" Y a esto Buda le plantea: "A esto, gran rey, le anuncio que la vejez y la muerte se nos vienen encima; cuando la vejez y la muerte se nos vienen encima, ¿qué debemos hacer?". Los invito a que consideren esa pregunta por unos momentos. Esta es la pregunta donde el estudio liberal empieza, pues esa es el tronco de toda reflexión sobre la razón de lo que significa ser humano. Esta pregunta "¿Qué se debe hacer?" es parecida a la que Sócrates hace al principio en La República, y este es el texto con el cual todos los estudiantes de segundo año en la Universidad de Columbia comienzan en el año escolar. La República abre con un debate entre Sócrates y Trasímaco sobre la naturaleza de la justicia. Trasímaco insiste en que la justicia es simplemente una función de la fuerza, una función del poder y aboga que quien tenga más fuerza define para todos lo que es justo y lo que es injusto. Así que, bajo esa teoría, la persona sensata, dice Trasímaco, se "comporta justamente" cuando es necesario o cuando quiere complacer a quienes tienen el poder, pero, de otro modo, ignorará las normas de justicia y se comportará como mejor le convenga.

Luego de proponerse esta teoría de justicia, un entendimiento que volvemos a ver en forma modificado en Maquiavelo (el cual sigue vigente hoy entre muchos pensadores políticos y entre muchos practicantes políticos), una vez que Trasímaco propone esta teoría de la justicia se prepara para irse y dejar la conversación, pero Sócrates se lo impide; Sócrates le pregunta forzosamente: ¿Te parece un asunto pequeño el determinar el modo de conducta al cual ateniéndonos cada uno podamos vivir más provechosamente nuestra vida? Esta es la pregunta fundamental de La República; esta es la pregunta angular de la educación liberal; es otra versión de la pregunta del Buda al rey Pasenadi: "Cuando la vejez y la muerte se nos vienen encima, ¿qué se debe hacer? Quiero aclarar que esta pregunta no es una pregunta filosófica. "¿Qué forma de vida vale la pena vivir?" es una pregunta que cada uno de nosotros debe contestar; nadie puede escaparse de ella y nadie nos la puede contestar; cada uno tiene que contestarla por sí mismo. Además, no es una pregunta que se puede contestar con recitar un credo o adoptar una religión; es una pregunta que hay que contestar en la práctica, es una cuestión de lo que los marxistas llaman praxis: ¿Cómo se vive bien? ¿Cuál es la vida buena?, y, ¿cómo la alcanzamos? Estas preguntas son tan amplias como la vida misma; son el tema del estudio de las artes liberales y constituye la plataforma sobre la cual desarrollamos todas las otras formas de estudios. O sea, las artes liberales nos ponen a pensar en cómo 
vivir para que cuando nos llegue la muerte, como dice Henry David Thoreau, no nos vayamos a dar cuenta de que nunca pudimos vivir.

\section{¿Y este tipo de preguntas pertenecen a la Academia?}

Los que abogamos por los estudios liberales como componentes imprescindibles en la educación universitaria, decimos enfáticamente que sí y decimos que tiene que estar en el centro de la educación universitaria y no a la periferia. Los grandes pensadores en todos los campos han conectado sus actividades a estas preguntas y, además, estas preocupaciones son las fuentes del genio, ya sea en el ámbito científico, técnico o estético.

Los orígenes de la educación liberal se remontan a la antigua Grecia, a una sociedad esclavista en donde se distinguió entre la educación apropiada para un esclavo - un sirviente - y la educación adecuada para el individuo libre, para el ciudadano. Hoy debemos hacernos la misma pregunta, o sea, si estamos brindando educación adecuada para un obrero profesional o la educación adecuada para un ciudadano libre.

Aristóteles escribe en su tratado sobre La Política, que "la ciudad más excelente no les concederá la ciudadanía a los obreros vulgares", pues dice él que es imposible ocuparse de asuntos virtuosos cuando se vive la vida de un obrero vulgar o de un jornalero, o sea, de un obrero contratado. Este es un sentimiento que hoy para nosotros no es aceptable, ya que vivimos en una sociedad donde no hay esclavos, donde consideramos el trabajo de jornalero como digno y debe ser obvio que esta persona debe tener derechos políticos. Así que, vivimos en una sociedad en la que Aristóteles no podría manejarse o imaginarse, una sociedad en la cual somos casi todos jornaleros y, al mismo tiempo, somos casi todos libres. Pero debemos reconocer que, aunque ser libre es bueno, sucede que la libertad trae sus propios problemas y dificultades; vivir libremente no es vivir al azar; la libertad no es la falta de orden; no es el movimiento, como decimos, "a lo loco", no es el caos; la libertad solo puede ejercitarse en un marco de orden. Lo que distingue a la libertad de la esclavitud no es la falta de orden, es la falta de arbitrariedad. Solo que el orden que caracteriza a la libertad es un orden autónomo, un orden autodirigido; este orden autónomo es difícil de lograr. Por ejemplo, queremos comer comidas que no son saludables y queremos no ejercitarnos. Tenemos conflictos en nuestros deseos y cuando se nos da la libertad de llevar a cabo nuestros deseos, con esto surge una serie de complicaciones, el orden autónomo, el orden libre es difícil y requiere ser cultivado; por eso, decía Aristóteles que este tipo de cultivo del hombre libre es necesario para el ejercicio propio de la ciudadanía.
Emmanuel Kant, un pensador mucho más cercano a nosotros, decía que las reglas que gobiernan a la naturaleza se llaman Física y que las reglas que gobiernan a la libertad de llaman Ética. La vida ética presupone la vida libre; o sea, la ética se refiere a las leyes que cumplimos libremente cuando estas no son impuestas desde afuera, y es algo intuitivo; nosotros entendemos intuitivamente que cuando hacemos el bien forzosamente, cuando se nos forza a hacer el bien, esa acción nos disminuye, acción por acción, y no tiene mérito, pero ocurre lo contrario cuando elegimos libremente llevarlo a cabo. Lo mismo decía Rousseau: "Ser manejado por los impulsos es esclavitud, mas la obediencia a las leyes que uno mismo ha prescrito es libertad".

\section{¿Este tipo de estudios es aplicable a la universidad moderna?}

El estudiante de hoy, el estudiante de 17 o 20 años que llega a la universidad, a pesar de los increíbles avances tecnológicos de nuestra época, no es muy diferente con respecto al autoconocimiento del estudiante de 17 o 20 años de hace 500 años, de hace 1,000 o 2,000 años. Ellos se enfrentan a los mismos dilemas existenciales, sienten en su carne el peso de preguntas perennes de los problemas fundamentales de la existencia humana, los cuales nuestros avances en el conocimiento no han podido resolver, y es a esta situación a la cual la educación general se dirige. Nos preguntamos: ¿Cómo podemos estimular y provocar en nuestros alumnos un desenvolvimiento interno? ¿Cómo cultivamos en nuestros alumnos un descubrimiento amplio de su propia humanidad? ¿Cómo transmitimos los valores e ideas que guíen en el futuro el uso del conocimiento profesional que también les damos a los estudiantes? ¿Cómo preparar ciudadanos no solo de un mercado libre, sino también de una sociedad democrática?

Dos tendencias simultáneas caracterizan la expansión del conocimiento en nuestra sociedad contemporánea. La primera tendencia es la híperespecialización, o sea, un estrechamiento y una concentración de la pericia en cuestiones cada vez más precisas y más técnicas. La segunda tendencia es hacia la complejidad: todo se ha vuelto más complejo; por ejemplo, ya no vivimos en el mundo de conflictos previsibles de la Guerra Fría; sin embargo, la textura de las tensiones internacionales es ahora mucho más compleja; el cambio climático es complejo, el Internet y las innovaciones en las tecnologías de información son disruptivas en maneras que no entendemos bien. Las implicaciones éticas de la manipulación genética humana están apenas empezando a amanecer entre nosotros. Donde quiera que miramos encontramos mayor conocimiento y mayor complejidad de lo que podemos asimilar racionalmente. Es debido a esta complejidad que la educación liberal 
es más necesaria y más importante hoy que nunca antes. Allí aprendemos a formar opiniones cuando las evidencias son inconclusas, hacer juicios que tomen en cuenta nuestros valores y nuestros prejuicios basados en nuestra subjetividad. La educación liberal es para desarrollar la facultad de juicio, aquella facultad en la cual dependemos cuando nos enfrentamos a dilemas que no pueden resolverse por la mera acumulación de datos o cálculos racionales. La educación liberal toma por dado que la educación técnica, la educación aplicada o profesional, no se presta a resolver las cuestiones humanas con las que vivimos a diario y en medio de las cuales nos desenvolvemos. O sea, tenemos que diferenciar entre la sabiduría y el conocimiento; esa distinción se remonta por lo menos a Platón que distinguió entre Téchne y Phrónesis. Aristóteles, discípulo de Platón, definió la phrónesis como sabiduría práctica o prudencia, o sea, juicio; lo cual tiene que ver tanto con el intelecto como con el carácter. Por otro lado, téchne se refiere a la técnica, a la destreza, al conocimiento aplicado. Recordemos la famosa frase de Sócrates: "La vida no examinada no merece ser vivida", phrónesis es lo que se necesita para vivir una vida examinada.

\section{¿Se contraponen los estudios liberales a la educación que solo valora el desarrollo del conocimiento técnico?}

En sus diálogos Platón identificó como uno de los mayores obstáculos a la vida examinada, el deseo de dominar y controlar; este deseo es hoy más fuerte que nunca y esto lleva a muchos a considerar el conocimiento técnico como el único tipo de conocimiento válido o valorable. La educación técnica es apropiada para objetos determinados, aquellos objetos que se encuentran en un campo claramente delineado; estos objetos pueden ser estudiados con un amplio grado de precisión, hasta podrían reducirlos a medidas exactas y expresarlos en forma matemática. La certeza del conocimiento matemático es lo que comúnmente se denomina verdadero conocimiento o conocimiento científico. $Y$ ya que los conocimientos técnicos pueden ser expresados con tanta precisión, también pueden ser transmitidos con facilidad a través de la enseñanza y, además, su precisión nos da el poder que deseamos sobre el objeto del conocimiento. Pero si el objeto que buscamos conocer es indeterminado, inherentemente impreciso, discutible, que no puede resolverse mediante la medida o el cálculo aritmético, entonces la situación es completamente diferente.

En el diálogo Eutifrón, Sócrates incluye entre tales objetos indeterminados: el bien, lo justo y lo bello; él atribuye esta resistencia a la definición precisa, esta resistencia al tipo de solución determinada que brinda el conocimiento técnico, a lo que Platón llama el "alma". Y en particular, estos objetos: lo bueno, lo malo, lo justo, lo bello no son susceptibles al conocimiento técnico por la capacidad que tiene el alma humana de la autoactividad, ya sea el automovimiento o la autorreflexión. Las artes liberales invitan a los estudiantes a la autorreflexión a hacerse preguntas difíciles sobre sus propias vidas.

\section{¿Por qué la Universidad de Columbia ha asumido con tanto ahínco los estudios liberales?}

En la Universidad de Columbia hemos visto que hacerse preguntas transcendentes cambia quiénes y cómo son nuestros estudiantes y este es precisamente el extraño carácter del alma autorreflexiva. La propia actividad de autorreflexión la transforma.

El método en que las artes liberales logran el autoconocimiento presume que el alma humana es algo más que un objeto estático disponible para el conocimiento técnico o el cálculo; este enfoque reconoce el carácter inherentemente elusivo de cómo somos y este objeto misterioso y resistente al determinismo del conocimiento técnico es el objeto de las artes liberales. Montaigne, gran pensador francés, escribió en uno de sus ensayos: "Nuestro deber es componer nuestro carácter, no componer libros, ganar batallas, ganar provincias, sino orden y tranquilidad en nuestra conducta. Nuestra gran obra maestra es vivir como se debe; todo lo demás: gobernar, acumular tesoros, construir, todo lo demás son solo pequeñeces". El conocimiento técnico no es suficiente para preparar al estudiante para el mundo en el cual este tendrá que tomar decisiones, el mundo actual.

La práctica liberal nos enseña a interpretar. La práctica liberal nos prepara para actuar cuando no sabemos. Como seres humanos, nosotros no producimos ni utilizamos el conocimiento en un vacío, sino dentro de una matriz de valores, creencias, intenciones, marco histórico. Por ejemplo, los instrumentos financieros de inversión y las instituciones que aumentan el flujo de capital son cruciales en nuestra sociedad, pero la única cuestión no es la de generar capital, la otra y la más difícil, es cómo reglamentar y regular el flujo de capital en una manera sostenible, o sea, de una manera justa y humana. En fin, no hay manera de escapar de la conclusión de que la educación, la verdadera educación, tiene que ver con la transformación personal, con aprender a cómo ser humano, con cultivar hábitos de autocrítica y una actitud abierta a lo nuevo y lo desconocido. Estos son los ambiciosos propósitos de la educación liberal.

¿Podría describirnos cómo han implementado los estudios liberales en la Universidad de Columbia y cómo logran los propósitos de promover en los estudiantes este pensamiento autorreflexivo de la vida examinada?

Para terminar, permítanme describir, rápidamente, cómo intentamos cumplir estos propósitos con el Core 
Curriculum en la Universidad de Columbia. El Core Curriculum es un conjunto de 5 cursos que todos los estudiantes a nivel de grado tienen que tomar, no importa qué carrera o especialización quieran seguir. Estos cursos son diferentes a otros que ofrece la Universidad en varias maneras. Tienen cuatro características que los distinguen:

1) La primera característica es que estos son cursos obligatorios y uniformes, o sea, todos los estudiantes toman el mismo curso, no un curso con el mismo nombre, sino el mismo curso donde leen los mismos textos y tienen el mismo tipo de enseñanza. Por ejemplo, todos los estudiantes de primer año en la Universidad de Columbia comienzan leyendo La llíada de Homero la primera semana de clases. El campus entero tiene 1200 estudiantes; todos los estudiantes cargan con una copia de esta obra que todos están leyendo; la tercera semana, después que llevan dos semanas con La llíada, todos al mismo tiempo proceden a La Odisea de Homero. Los estudiantes de segundo año, como lo mencioné, comienzan el año escolar leyendo $L a$ República de Platón; luego de leer esta obra, leen La Política de Aristóteles y La Ética de Aristóteles. Estos cursos son obligatorios y uniformes. Esta es la primera característica de los cursos del Core Curriculum.

2) La segunda característica es que solo estudiamos textos primarios o textos originales, no leemos artículos sobre Platón, leemos a Platón; no leemos interpretaciones del Corán o del Nuevo Testamento o de Nietzsche o Freud, leemos el Corán, el Nuevo Testamento, a Nietzsche, a Freud. Este énfasis en obras importantes de la historia literaria está en el centro de estos cursos; esta es la segunda característica: nos enfocamos en trabajos originales. Otra nota sobre esta característica es que esto convierte a esas clases en no disciplinarias, no son clases de literatura, no son clases de historia, no son clases de filosofía, no son clases de ciencias políticas, no son clases de sociología, aunque en todas estas áreas se tratan esos temas y esos textos son las fuentes de donde surgieron esas disciplinas; así que este énfasis en trabajos originales hace que estos cursos sean predisciplinarios o metadisciplinarios.

3) Esto nos lleva a la tercera característica de los cursos Core, son seminarios enfocados en el debate y la conversación, o sea, son clases pequeñas, un máximo de 22 estudiantes, así que, la clase de primer año que tiene 1200 estudiantes, para acomodarlos en estos salones pequeños, en estos grupos pequeños, necesitamos 60 secciones o más. El curso del primer año del Core Curriculum que se llama Literature Humanities tiene 62 secciones este semestre. Esto se convierte en una práctica importantísima, seminarios basados en discusión, porque lo que estamos enseñando al estudiante es un conjunto de conocimientos específicos en debate de grandes ideas y grandes temas de la historia de la humanidad. Esto no se puede hacer con la memorización y con aprendizaje bruto. Esto solo se puede hacer con análisis, con síntesis, con cuestionamientos, con debates en conversación. Así en todas las clases con grupos máximos de 22 estudiantes se implementa esta misma metodología; esa es la tercera característica.

4) La cuarta característica es la interdisciplinariedad de estos cursos. Para montar estos cursos tenemos profesores de todos los departamentos de la Universidad. Tenemos sociólogos, antropólogos, historiadores, filósofos, científicos, políticos, críticos literarios, etc. Todas las áreas de las humanidades y las ciencias sociales vienen a dar estas clases, así que, este es un programa interdepartamental.

Estos cursos son comunes, dos de ellos son de un año entero y lo voy a describir rápidamente. El primer curso se llama Obras maestras de la Literatura y la filosofía occidental; es un curso que dura un año entero y como dije, empieza leyendo a Homero y también leen a Sófocles, Eurípides, los dramaturgos de la antigua Grecia, los historiadores; inclusive leemos La Eneida de Virgilio, el Nuevo y el Viejo Testamento.

Todo eso se lee en el primer semestre. En el segundo semestre empiezan con Paraíso Perdido de Milton y leen también a Don Quijote, a Dante, a Shakespeare, a Virginia Wolf, Dostoyevski y otros. Los mismos estudiantes tienen el año entero al mismo profesor haciendo este recorrido de las obras maestras y fundamentales de la literatura occidental.

En el segundo año, los estudiantes toman un curso parecido que se llama Introducción a la civilización contemporánea de cultura occidental y este es básicamente un curso de pensamiento ético y religioso. Comenzamos con La República de Platón; seguimos con Aristóteles; leemos los filósofos antiguos históricos; leemos también textos bíblicos; leemos a Maquiavelo, Hobbes, Alá. En el segundo semestre leemos a Marx, a Freud, a Nietzsche, a Dubois, a Fanón, a Foucault; de nuevo son cursos de un año entero para todos los estudiantes de segundo año; no importa qué carrera vayan a tomar y están en el mismo grupo de estudiantes, con el mismo profesor el año entero. Las otras tres clases son solo de un semestre, en vez de clases de un año entero. Una de ellas se llama Obras maestras del arte occidental. Aquí se estudian obras como el Partenón de Grecia, con la cual iniciamos; se estudia escultura, pintura y arquitectura, así como también catedrales, templos, obras maestras de la pintura, la escultura en la tradición occidental, con el mismo formato. Son estudios de trabajos originales, la mayoría 
de veces en fotos, aunque incluimos visitas a los grandes museos de New York. También permanecen los grupos pequeños y la misma estrategia de debates y discusión.

El cuarto curso es un curso de música, se llama Obras maestras de la música occidental, y allí estudiamos obras maestras de la música comenzando con los Cánticos Gregorianos de la Edad Media y estudiamos los grandes compositores; grandes obras barrocas, clásicas, del Renacimiento, hasta la música moderna experimental. Estos cursos los toman todos los estudiantes. Y el último curso se llama Fronteras de la Ciencia, es un curso donde buscamos que nuestros estudiantes aprendan el pensamiento científico, es decir, cómo piensan los científicos, qué tipo de preguntas se hacen y cuáles métodos se usan para contestar estas preguntas.

\section{¿Qué tiempo tiene de establecido este programa?}

Nuestro Core Curriculum, con los cinco cursos que hemos descrito, requerido a todo estudiante de Columbia, representa nuestro compromiso con el ideal del pensamiento liberal.

Este no es un experimento nuevo; nosotros en Columbia Ilevamos haciendo este Core Curriculum desde el año 1919, así que vamos a cumplir ya 100 años. Todo estudiante que estudió en Columbia tomó estos cursos; estudió esos libros y cada vez que se reúnen cualquier graduado de Columbia con otro de nuestros egresados tienen esta experiencia en común; tienen este vocabulario, tienen esta base de datos y conocimientos y experiencias que pueden compartir.

Yo mismo estudié a nivel de grado en la Universidad de Columbia, habiendo emigrado a Estados Unidos a la edad de 12 años y este currículum; esta educación general, clásica, literalmente transformó mi vida y me llevó a la carrera como académico, profesor y ahora director del mismo programa. Este currículum tiene un impacto fundamental en nuestros estudiantes; tiene un impacto fundamental en la institución, en el profesorado. $Y$ yo espero que esta presentación del programa de Columbia y de mis reflexiones sobre la historia y la filosofía educativa que informa la organización de nuestro programa, sirva de inspiración, sirva de, hasta cierto punto, modelo o de fortaleza para implementar con los estudiantes y en el currículo. Promovemos programas educativos que se fijan en el desarrollo intelectual, pero también en lo espiritual, personal y social de los estudiantes. Apostamos a que las universidades logren organizar programas para preparar ciudadanos libres para gobernar a través de la participación de un mundo y un futuro mejor. En eso estamos, esa es nuestra misión desde Columbia.

Hay una elevada preocupación por la formación técnica que desplaza la formación ética y ciudadana. ¿Qué más se puede hacer para lograr esta batalla?
Sí, es cierto. Es una de las batallas que tenemos que tomar y, en ese sentido, abogar por las artes liberales es algo contracultural; es decir, estamos nadando contra la corriente, pero esto solo enfatiza la importancia de esta labor. Tenemos como lección a Alemania, el país que desarrolló la universidad contemporánea, la universidad dedicada a la maestría del conocimiento técnico. Fue este mismo país y bajo este mismo sistema universitario que nos dio el régimen Nazi, el cual fue capaz de cometer las atrocidades más espeluznantes del siglo XX. Y esto nos ilustra que el conocimiento por sí solo es peligroso; es decir, que nos puede llevar a la deshumanización de nuestra práctica técnica. Así que, aunque sí es una lucha en contra de la corriente, es una lucha por el alma de nuestros tiempos, el alma de nuestra contemporaneidad. Y los retos a los cuales nos vamos a enfrentar, que ya empezamos a enfrentarlos, son retos que tienen que ver con el cambio del medioambiente por la actividad humana, cambios que tienen que ver con la tecnología, como la introducción de los autos sin chofer, los autos que se manejan solos, la automatización de las industrias, la modificación genética, no solamente en las especies animales y vegetales, sino también de los propios humanos. Todos esos son retos que están a la puerta, que ya tenemos que tomar acciones y decisiones sobre ellos. Así que, hoy en día una educación basada en valores es una educación que nos invita a reflexionar sobre el bien humano, la cual es más necesaria que nunca.

Es más fácil implementar este tipo de estudio en una sociedad desarrollada y con los problemas de subsistencia resueltos. Sin embargo, ¿cómo pueden los jóvenes de países subdesarrollados dedicar tanto tiempo a la autorreflexión cuando su prioridad es aprender conocimientos técnicos para poder ganar dinero con inmediatez?

Sí, sí. En realidad, tenemos que admitir que es lo normal y lo natural que un joven de hoy se interese más que todo en la tecnología y más que todo en la acumulación de riquezas porque vivimos en una sociedad en donde los valores capitalistas son ascendentes; ellos son los valores más resaltados en el discurso público, en los medios de comunicación, en las películas, etc. Si nosotros estuviéramos en esa situación, quizás si fuéramos nosotros hoy chicos de 16, 17 o 18 años tuviéramos una mentalidad parecida; es decir, es normal. Es como si se les ofrece un vaso de agua sucia, agua con lodo y ellos tiene sed, pero esa es la única agua que les ofrecemos. Ellos se la van a beber. Pero si nosotros les ofrecemos además de esa agua sucia un vaso de agua limpia, ellos elegirán el agua limpia. Así que, nosotros tenemos que ofrecerle a nuestra juventud un vaso de agua limpia; ofrecerle a nuestra juventud una visión educativa, alternativa y como ya he enfatizado, no es en vez del entrenamiento técnico, sino como base o como suplemento del otro lado al entrenamiento técnico. Los jóvenes quieren, tiene sed 
de estas preguntas; estas cuestiones les interesan profundamente y nos toca a nosotros como ejemplo, como profesionales, presentarles un modelo de nuestra propia vida, de nuestro propio discurso, de nuestro propio comportamiento. A mi parecer, el profesorado, especialmente el profesorado en estudios liberales, el profesorado de estudios generales, tiene un llamado de la misma manera que el sacerdocio; es un llamado que no solo tiene que ver con cómo uno va a transmitir cierto conocimiento, sino de cómo uno va a vivir una vida que demuestre ese conocimiento, que demuestre esa práctica liberal. Es decir, nosotros en los estudios liberales estamos hablando y enseñando el significado de la libertad humana, con nuestro comportamiento, con nuestro discurso, con nuestra forma de ser; nosotros podemos demostrar el tipo de vida que se puede vivir basado en estos valores, basado en esta práctica de indagación, de escudriñamiento, de preguntar, de tener integridad intelectual y no estoy hablando de una vida religiosa. Uno puede pertenecer a un credo o asistir a una iglesia o no; uno puede ser practicante del cristianismo o no; uno puede ser ateo; uno puede ser budista. No estoy hablando de una religión, sino de una vida humana completa. Una vida humana alerta, una vida humana que respeta la libertad, la verdad y los valores que nos hacen humanos.

Es admirable cómo la Universidad de Columbia ha mantenido su programa durante casi 100 años, pero nos gustaría preguntar: ¿Con qué frecuencia revisan su programa y si solo incluyen estos textos de las culturas clásicas o podrían incluirse otros textos ancestrales y de tanta valía como los de la literatura occidental?

$\mathrm{Si}$, muy bien. Es una pregunta muy certera. El programa de Columbia comenzó, como dije, hace casi cien años, cuando no teníamos acceso como lo tenemos hoy a otras tradiciones intelectuales. Creo que si el programa se estuviera armando en el día de hoy, buscaríamos incluir otras grandes tradiciones intelectuales, filosóficas, artísticas. Pero podemos cualificar eso y debo decir que en el programa de Columbia, además de este programa, requerimos que cada estudiante tome dos cursos en tradiciones no occidentales; hay una gran variedad de esos cursos; hay cerca de 60 cursos y ellos escogen dos de ellos. Así que, tratamos de incluir en la educación del estudiante esas otras elecciones. Como lo hacemos aquí en la Universidad de Columbia, admitimos que no es la única manera de hacerlo. Yo conozco y he participado en la creación de otros programas que incluyen otras tradiciones y me parece que son excelentes, pero voy explicar un poco la justificación de los dos hilos que sostienen nuestro programa: el hilo con énfasis en lo clásico y el hilo de la tradición occidental.

Primero en cuanto a los textos clásicos, nosotros tenemos el objetivo de presentarle al estudiante los textos que han sido más importantes y fructíferos en la investigación, en la reflexión filosófica, literaria, artística de las grandes cuestiones humanas; o sea, eso quiere decir que vamos a ir desde lo más antiguo que podamos, que en el caso de la literatura estamos hablando de, así certeramente, a la Grecia antigua. Antes de eso tenemos fragmentos e indicios, pero los primeros trabajos literarios completos que tenemos son de la Grecia antigua. Así que, si nos remontamos a lo más antiguo que podamos ir y nos preguntamos cuáles han sido los textos, las intervenciones que han sido más controversiales, que han contribuido más a cómo entendemos hoy el mundo, las categorías que usamos, el vocabulario que usamos, las instituciones que tenemos; esos textos van a darle al estudiante las herramientas para poder entender el mundo contemporáneo a través de entender cómo surgió el mundo contemporáneo. Es decir, entendemos que el presente surge del pasado y que para entender el presente más efectivamente y poder intervenir e influenciar el presente, lo podemos hacer más efectivamente con un conocimiento sólido del pasado, lo cual nos va a llevar a los clásicos obligatoriamente.

Hay un gran valor en introducir esos textos antiguos porque le da al estudiante una noción de cuáles son las cosas que se mantienen igual desde hace dos mil años y cuáles son las cosas que son diferentes de hace dos mil años en cuanto a la actividad humana. Cabe decir que, si creamos un curso en Latinoamérica, debe dárseles énfasis a otros textos, como los diarios de Colón y los documentos de la organización colonial; luego, el surgimiento de los movimientos de independencia, etc. Creo que un curso en Latinoamérica les daría mayor atención a estos temas y que probablemente los Estados Unidos no se lo daría.

El segundo hilo es el occidental, hay un gran valor en enseñar una tradición intelectual; es decir, si nosotros leemos a Platón, leemos a Aristóteles, quien está en diálogo con Platón, y leemos a San Agustín, quien está en diálogo con Platón, y a Santo Tomás de Aquino, quien está en diálogo con Aristóteles, y a Dante, quien está en diálogo con todos ellos, y luego a Shakespeare y a Cervantes. En esa tradición tenemos una larga conversación y esta es una de las grandes riquezas de poder enseñar con el hilo occidental; lo mismo podría hacerse con un hilo del oriente. Hay un gran valor en este método de enseñar a la humanidad a través de una tradición que es coherente y que conlleva a una conversación, a unos temas, unas inquietudes que autor tras autor y artista tras artista ha tratado de enfrentarse a ella. Así que, hay muchas maneras de hacerlo. Creo que el énfasis en lo clásico y el énfasis en hacer una tradición coherente tiene mucho valor. Así que, se puede hacer de varias maneras, pero hay mucho valor en hacerlo con el hilo de una misma tradición y con énfasis en los clásicos. 